\title{
Una revisión crítica de la Ley de Bradford
}

Rubén Urbizagástegui Alvarado Bibliotecario Asociado, Biblioteca Tomás Rivera, Universidad de California, Riverside, P.O.Box 5900, Riverside, CA 92507, Estados Unidos de Norteamérica.

E-mail: ruben@ucrac1.ucr.edu

\section{RESUMEN}

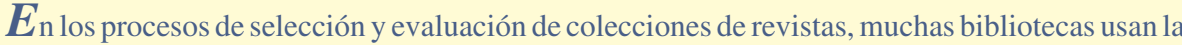
ley de Bradford para identificar las revistas más productivas. Sin embargo, debido a que la ley de Bradford establece un corte en el tiempo, existe la posibilidad de que en ese periodo, existan revistas con diferentes años de participación y con diferentes frecuencias de publicación. De modo que es posible que no todas las revistas tengan la misma oportunidad de publicar artículos relevantes sobre el asunto conforme a lo establecido por la ley de Bradford. El objetivo de este trabajo es investigar esas suposiciones. Los resultados muestran que existe una alta correlación entre la frecuencia de publicación, los años de participación y la productividad de una determinada revista. También la regresión lineal muestra que hay una relación de causa-efecto entre esas variables. Se ofrece un modelo alternativo basado en un índice estandarizado de la productividad, que parece ser más adecuado para identificar revistas que al mismo tiempo sean especializadas en el área y más productivas.

\section{ABSTRACT}

In the selection and evaluation process for collection development, many libraries use Bradford's law to identify the most productive journals. However, because the Bradford's law establishes a cut in a time-span, there is a possibility that along the time-span journals with different years of participation and different frequencies of publication will occur. So, it is possible that not all the journals will have the same opportunity to publish relevant articles on the subject as stated by the Bradford's law. The objective of this research is to demonstrate these assumptions. The findings shows that there is a high correlation between frequency of publication, years of participation, and productivity of a given journal. The linear regression also shows that there is a cause-effect relationship between these variables. An alternative model based on a standardized index of productivity prove to be more adequate to identify journals that at the same time are both devoted to the area and more productives.

\section{PROBLEMA DE INVESTIGACIÓN}

T a productividad científica de las reLvistas ha sido estudiada frecuentemente a partir de la metodología propuesta por Bradford (1934) y ahora en el área de la Bibliotecología y Ciencias de la Información es usada y conocida como la "Ley de Bradford". Bradford originalmente estableció esta ley afirmando que:

Si las revistas científicas fuesen ordenadas en forma decreciente de productividad de artículos en un determinado asunto, pueden ser divididas en un núcleo de revistas más particularmente dedicadas al asunto y varios grupos o zonas que contengan el mismo número de artículos que el núcleo, pero el número de revistas en el núcleo y en las zonas sucesivas serán ordenados como $1: n: n^{2} \ldots$
Donde $1: n: n^{2} \ldots$ representan las revistas incluidas en el núcleo y las zonas sucesivas. De este modo, todas las revistas actúan como una familia de generaciones sucesivas de parentesco disminuyente, con cada generación subsecuente necesitando un número mayor de revistas para producir la misma cantidad de artículos. Esto significa que el número de revistas necesarias para obtener la misma cantidad de artículos crece exponencialmente en la distribución, mientras que en la siguiente generación es necesario incluir más revistas menos productivas para obtener igual número de artículos. Como podemos ver, la ley de Bradford es simplemente la descripción de una relación cuantitativa entre las revistas y los artículos científicos contenidos en una bibliografía sobre un asunto determinado. Esta ley trata de demostrar que en la producción de artículos en las revistas existe una distribución altamente desigual donde la mayoría de los artículos está concentrado en una pequeña población de revistas, mientras que una pequeña proporción de artículos se dispersa sobre una alta cantidad de revistas. En el proceso de concentración, la primera zona llamada el "core" o núcleo, debería contener las revistas dedicadas al asunto de la literatura estudiada. Para los propósitos de adquisición en una biblioteca, la zona uno o núcleo debería identificar aquellas revistas con capacidad de publicar el mayor número de artículos dedicados al asunto y pueden servir como guía para establecer las políticas de 
adquisición y las estrategias de desarrollo de colecciones, conforme a lo sugerido por Goffman \& Morris (1970). De acuerdo con Brookes (1969), para una adecuada aplicación de la ley de Bradford a la bibliografía en análisis le deben ser impuestas cuatro condiciones: 1) el asunto de la bibliografía debe estar bien definido; 2) la bibliografía debe ser completa, esto es, todos los artículos y revistas relevantes deben estar incluidos en la bibliografía; 3) la bibliografía debe ser de un periodo limitado, de modo que todas las revistas contribuyentes tengan la misma oportunidad de colaborar con artículos; y 4) el número de artículos contenidos en el núcleo (zona uno) no puede ser inferior a la mitad del número de revistas que produce un solo artículo.

Sin embargo, como la ley de Bradford establece un corte en el tiempo conforme a lo establecido en la condición 3, a lo largo del periodo de investigación, es posible encontrar revistas con diferentes años de participación y diferentes frecuencias de publicación. De modo que, es posible que no todas las revistas tengan la misma oportunidad de publicar artículos relevantes sobre el asunto, conforme a lo establecido por la ley. Esto puede ocurrir debido a que algunas revistas pueden haber nacido o perecido al comienzo, al medio, o al final del periodo de estudio. Aquellas revistas nacidas o cesadas en algún periodo de la investigación tendrán menos oportunidades de publicar que aquellas que hayan participado en el periodo entero. Igualmente, la frecuencia de publicación de la revista juega un factor importante. Una revista mensual tendrá mayores posibilidades de publicar más artículos que una cuatrimestral, una semestral o una revista anual. Estos hechos muestran claramente que el periodo de participación y la frecuencia de publicación de la revista, tendrán alguna influencia en la productividad de esa revista. Por lo tanto, si se quiere comprender mejor el fenómeno de la distribución decreciente de artículos en revistas científicas, las siguientes preguntas deberían ser respondidas:

¿La frecuencia de publicación de una revista influye en su productividad y por tanto en su rango en el modelo de Bradford?

¿ ¿El número de años con que una determinada revista participa en el periodo de la investigación, influye en la productividad de esa revista y por tanto también en su rango en el modelo de Bradford?

¿ $\mathrm{Si}$ estas dos condiciones son verdaderas, es posible desarrollar un modelo alternativo que tome en consideración tanto el número de años de participación y la frecuencia de publicación en la medición de la productividad de una revista?

¿ ¿Este modelo alternativo identificaría efectivamente el núcleo de las revistas más productivas en el área en estudio?

Las respuestas a estas preguntas serían útiles para evitar la distorsión en las políticas de adquisición y desarrollo de colecciones en una biblioteca. Si el periodo de participación y la frecuencia de publicación afectan la productividad de una revista, estas variables también pueden influir su colocación en el tope o al final del rango en el modelo bradfordiano. En consecuencia, la ley de Bradford podría tener la habilidad de identificar las revistas más productivas, pero no necesariamente las más dedicadas (o especializadas) en el asunto de la literatura estudiada. Esta particular situación podría ocurrir como consecuencia de que el modelo, al estar basado en un periodo específico, hace competir en igualdad de condiciones, la productividad de revistas con diferentes frecuencias de publicación y con diferentes años de participación en el periodo investigado, y con eso, homogeniza lo que es naturalmente heterogéneo, i.e. convierte en estático lo que es dinámico por naturaleza, pero aún ignora esta dinámica. La aplicación mecánica de la ley de Bradford, sin considerar los factores señalados anteriormente, puede traer consecuencias infortunadas para las bibliotecas, ya que como fue muy bien indicado por Pinheiro (1982) y Urbizagástegui (1983, 1985), si la ley de Bradford es usada como base para las políticas de adquisición puede inducir a las bibliotecas a adquirir revistas que no solamente no están dedicadas al asunto estudiado, sino que también son señaladas de una manera falsa como altamente productivas.

\section{REVISIÓN DE LA LITERATURA}

A pesar de que algunas experiencias empíricas parecen haber verificado la clásica aplicación de la ley de Bradford a algunas disciplinas tales como las cien- cias de la computación (Kendall, 1960), geografía (Aiyepeku, 1977), bibliotecología (Bulick, 1978), y agricultura (Lawani, 1973), "el suceso alcanzado hasta ahora en la explicación y fundamento teórico de la ley de Bradford y fenómenos correlativos pueden ser evaluados como modestos" (Oluic-Vukovic, 1993). Sin embargo, es necesario apuntar que los autores citados anteriormente no controlaron la frecuencia de publicación ni el periodo de participación de las revistas estudiadas. Además, otras investigaciones han obtenido resultados contradictorios. Por ejemplo, para identificar el núcleo de revistas sobre conservación y preservación de materiales bibliotecarios, De Pew \& Basu (1986) analizaron los artículos indizados en Library Literature y Library and Information Science Abstracts. Todos los fascículos de esas dos fuentes fueron investigados desde enero de 1978 hasta diciembre de 1984 bajo los encabezamientos de materia Conservación y Preservación y sus referencias cruzadas, pero solamente los items publicados entre 1979 y 1982 fueron considerados para el estudio. Las revistas fueron agrupadas en tres zonas que contenían 5, 27, y 135 revistas, respectivamente. Los autores encontraron que 2 revistas (de un total de cinco) del núcleo no tenían relación con tópicos de conservación ni preservación.

También observaron que algunas revistas dedicadas a preservación tales como Conservation Administration News fueron ordenadas en la segunda zona, y otras revistas altamente productivas simplemente no aparecieron en ninguna zona. Baguena Cervellera (1984) también mapeó las revistas médicas españolas de microbiología entre 1801-1900, y encontró un total de 27 revistas que produjeron 438 artículos. La dispersión de Bradford mostró 2, 5, y 20 revistas en el núcleo y zonas 2 y 3 , respectivamente. Sin embargo, ninguna de las revistas colocadas en el núcleo ni en la zona 2 estaban dedicadas a microbiología. El área estaba enterrada en el campo más general de medicina. La autora explicó la ausencia de revistas dedicadas a microbiología, por la falta de institucionalización de este campo científico. Figueiredo (1973) estudió la literatura de geología de 1960 a 1965 en el Brasil y encontró 10 revistas en las zonas 1 a 3 , con solamente 4 de 

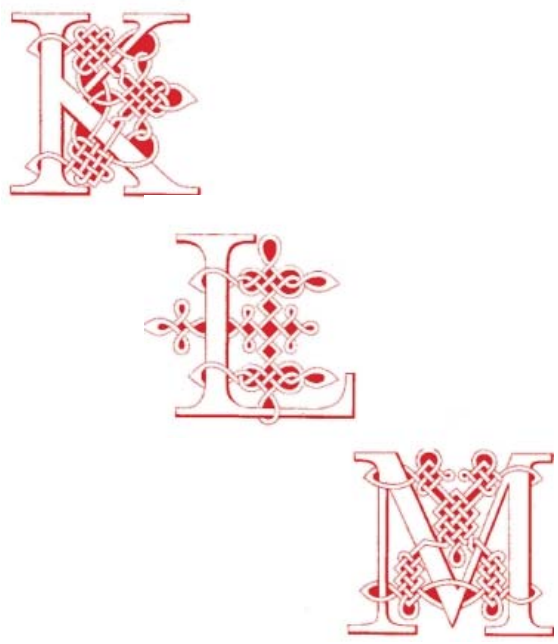

"Esas muestras tomadas de la literatura hacen evidente que la ley de Bradford es un indicador defectuoso de la productividad, ya que no considera la frecuencia de publicación ni el número de años con que una revista participa en el periodo de la investigación. Por eso, es necesario buscar métodos alternativos para medir la productividad como una herramienta para la toma de decisiones en la adquisición.”
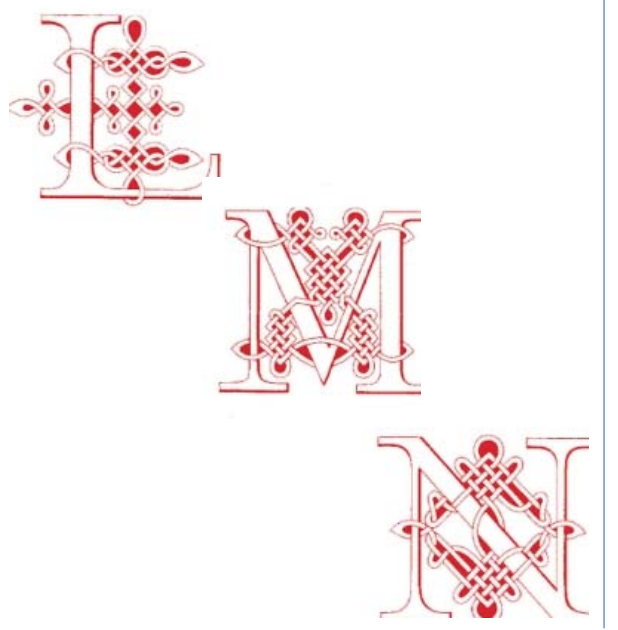

ellas dedicadas a geología. Queiroz (1975) y Caldeira (1975) observaron padrones similares en las áreas de botánica y agricultura donde el núcleo incluyó algunas revistas no dedicadas a esos campos. Similarmente, Pao (1979) encontró que a pesar de que los datos en musicología computarizada satisficieron las condiciones establecidas por la ley de Bradford, el núcleo de la distribución identificó 3 revistas diferentes, ninguna de las cuales estaba dedicada a musicología. Las 12 revistas más productivas estaban enterradas en una variedad de asuntos tan distintos que iban desde ingeniería hasta investigación musical.

Esas muestras tomadas de la literatura hacen evidente que la ley de Bradford es un indicador defectuoso de la productividad, ya que no considera la frecuencia de publicación ni el número de años con que una revista participa en el periodo de la investigación. Por eso, es necesario buscar métodos alternativos para medir la productividad como una herramienta para la toma de decisiones en la adquisición. En adición, algunos científicos de la información han estado últimamente más preocupados con la formulación matemática de la ley, y aún insisten en discutir si esta formulación matemática se ajusta a la observación gráfica o aun si tiene un carácter científico (Vickery, 1948; Brookes, 1969). Otros autores han propuesto variaciones en el modelo, tales como la distribución jerárquica (Basu, 1992), mientras que otros han desarrollado rangos sectorizados (Sivers, 1987). Más aún, algunos autores han estudiado el análisis empírico de los modelos de la ley de Bradford (Qiu, 1990). A pesar de que esas curiosas discusiones son necesarias para los académicos, no ofrecen a los bibliotecarios justificaciones suficientes para luchar contra el recorte en los presupuestos dedicados a las suscripciones. Los bibliotecarios estamos más preocupados con la identificación de revistas más productivas y menos preocupados en el ajustamiento a modelos matemáticos o sus variantes.

\section{MODELO TEÓRICO}

El punto central de esta investigación es estudiar las influencias, que la frecuencia de publicación y el periodo de participación en el periodo de la investigación tienen en la productividad de una revista.
Tomando la productividad de las revistas como la variable básica, se pueden formular algunas proposiciones para ser investigadas y analizadas en relación con las otras variables conforme a las propuestas en el siguiente modelo teórico:

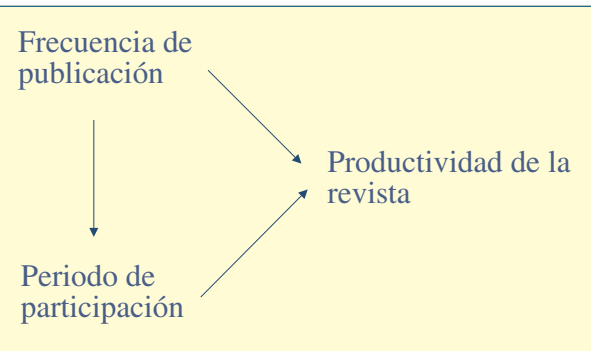

Para alcanzar los objetivos de la investigación, las variables envueltas en el modelo teórico se definen como sigue:

\section{Productividad de la revista}

Esta variable dependiente se refiere a la cantidad de artículos producidos por una determinada revista durante el periodo de investigación. Se supone que en un determinado año, cualquier revista produce una cierta cantidad de artículos, y que algunos o todos ellos están dedicados al asunto estudiado. Por eso, es posible verificar la existencia de revistas de alta productividad, de productividad media, y de baja productividad. Esta investigación verificará este padrón, cuantificará y medirá su relación con las variables independientes.

\section{Periodo de participación}

Esta variable independiente se refiere al número de años con que una determinada revista participa en el periodo de la investigación. Se supone que una investigación del tipo de la ley de Bradford ocurrirá en un periodo determinado. Cuando este periodo es determinado, incluirá revistas con diferentes años de participación, ya sea con uno, dos, tres años, y así sucesivamente, mientras que otras revistas participarán en todo el periodo de la investigación.

\section{Frecuencia de publicación}

Esta variable independiente se refiere al intervalo con que una determinada revista es publicada. Las revistas son publicadas cada semana (semanal), dos veces por semana (quincenal), una vez al mes (mensual), cada dos meses (bimestral), cada tres meses (trimestral), cada cuatro 
meses (cuatrimestral), dos veces al año (semestral), o una vez al año (anual). Debe considerarse que el número de fascículos producidos variarán de acuerdo con la frecuencia de publicación de la revista. Por ejemplo, una revista mensual producirá más fascículos que una anual; de este modo, aumenta sus posibilidades de producir más artículos y por tanto de colocarse en el tope de la distribución Bradford. Por ejemplo, en un periodo de cinco años, una revista mensual puede producir 60 fascículos, mientras que una anual producirá solamente 5; y, aun si la revista anual produce 10 artículos por fascículo, acumulará solamente 50 artículos en los cinco años, mientras que si la revista mensual produce solamente 1 artículo por fascículo acumulará 60 artículos en el mismo periodo.

\section{HIPÓTESIS}

Se supone entonces que una revista publicada con más frecuencia tendrá mayor oportunidad de producir más artículos que una revista de menos frecuencia. Más aún, una revista que participa con más años en el periodo de la investigación tendrá también más posibilidades de producir más artículos que una revista que participa con menos años en el mismo periodo. En consecuencia, es posible formular la hipótesis de investigación del modo siguiente:

La frecuencia de publicación de una revista y el número de años con que participa en el periodo de la investigación influyen en la productividad de la revista, de tal modo que, cuanto mayor su frecuencia de publicación, mayor su productividad y cuanto mayor su participación en el periodo de investigación, mayor también su productividad; y viceversa.

\section{DISEÑO DE LA INVESTIGACIÓN}

Para analizar la importancia del periodo de participación y la frecuencia de publicación en la aplicación de la ley de Bradford, el campo de la sociología fue escogido como área de experimentación. El periodo escogido fue de 1987 a 1991, debido a que una búsqueda aleatoria en la base de datos SocioFile produjo cero artículos para los años 1992-1993. Esto significaba que hasta diciembre de 1993, SocioFile todavía no había indizado los fascículos de la mayoría de las revistas producidas entre 1992 y 1993, indicando así que la indización de esas revistas to- davía estaba en procesamiento. SocioFile, la versión CD-ROM de Sociological Abstracts and Social Planning/Policy Development Abstracts, contiene citas y resúmenes de artículos de más de 1,600 revistas del área de ciencias sociales. Para identificar las revistas en el área de sociología, fue usada la base de datos de revistas del sistema Melvyl de la Universidad de California. La base de datos de revistas del sistema Melvyl contiene 742,438 títulos de revistas, representando $1.228,100$ copias de la Universidad de California, la Universidad de Stanford, la Universidad del Sudeste de California, las bibliotecas de la Universidad del Estado de California, el Centro Getty para la Historia del Arte y las Humanidades, y la Academia de Ciencias de California. Para identificar las revistas de sociología en la base de datos Melvyl, fue usada la siguiente estrategia:

Find Indexed by Sociological abstracts and Sociology or Social Sciences

Este proceso produjo 225 récords representando revistas del mundo entero. Para identificar solamente las revistas producidas en los Estados Unidos, fue realizada una revisión individual de esos 225 récords para eliminar cualquier registro duplicado/triplicado de la misma revista. También fueron eliminadas las revistas que habían cesado en su publicación antes del periodo designado para esta investigación (1987-1991) o habían comenzado a ser publicadas después de este perio- do. Este recorte produjo una lista de 103 revistas. Entonces, suponiendo que esas 103 revistas podrían no ser todas publicadas en los Estados Unidos, fue realizada una revisión de aquellas aparecidas en el Ulrich International Directory, 1993-1994 (1993). Finalmente la lista de Ulrich fue mezclada con aquellas identificadas en la base de datos de revistas Melvyl. La lista consolidada produjo 155 títulos. Sin embargo, 11 de esos títulos fueron eliminados debido a que eran series monográficas antes que revistas. También fueron eliminados 29 títulos que no pudieron ser identificados en ninguna base de datos. Sin una verificación completa de sus registros sus productividades no podrían ser contadas. Este proceso dejó una lista final de 115 revistas. Para levantar la productividad de artículos de las revistas entre 1987-1991, fue realizada una búsqueda individual en SocioFile, PsychLit, ABI Inform, Current Contents, y Firstsearch. Pensando que tal vez alguna revista extranjera podría tener el mismo título que una americana, fue usada la siguiente estrategia:

[nombre de la revista] in jn and cp=United States

Después, la búsqueda de la productividad fue conducida año por año para cada revista. Para controlar la frecuencia de publicación y el periodo de participación de cada revista fue construida una escala. Esta escala tomó en consideración el número de años del periodo de investigación y la frecuencia de publicación que se esperaba identificar para cada revista. Se supuso que una revista semanal podría publicar hasta 52 fascículos en un año, hasta 104 en dos, hasta 156 en tres, 208 en cuatro y 260 fascículos en cinco años. Igualmente una revista mensual podría publicar hasta 12 fascículos en un año, hasta 24 en dos, 36 en tres, 48 en cuatro, y hasta 60 en cinco años. La misma lógica fue usada para las revistas bimestrales, trimestrales, cuatrimestrales, semestrales, y anuales. La lógica de este proceso está resumida en la Tabla 1.

\section{RESULTADOS}

De las 115 revistas de sociología identificadas como productoras de artículos entre 1987 y 1991,9 revistas (5.8\%) participaron solamente con un año; $14(9 \%)$ con dos; $6(3.8 \%)$ con tres; 9 (5.8\%) con cuatro; y $77(49.7 \%)$ con cinco años, en el periodo de la investigación. Esto claramente significa que no todas las revistas participaron homogéneamente en el periodo indicado para la investigación. Por ejemplo, Critical Sociology comenzó a ser publicada en 1988 como continuación de The Insurgent Sociologist que desapareció en 1987. De aquí que ambas participaran en el periodo de la investigación, pero con diferente cantidad de años. Simultation and Games desapareció en 1989, y fue sustituida por Simulating and Gaming que comenzó en 1990. Advances in Medical Sociology comenzó a ser publicada en 1990; en consecuencia, su participación fue solamente de dos años. Political Anthropology desapareció en 1989, mientras que Research in Social Work Practices comenzó en 1991. Perspectives in Social Problems desapareció en 1989, mientras que Responsible Community $\mathrm{y}$ Research in 
Tabla 1

Número de fascículos esperados por revista según frecuencia de publicación y años de observación

\begin{tabular}{|l|l|l|l|l|l||}
\hline \multirow{2}{*}{$\begin{array}{c}\text { Frecuencia de } \\
\text { Publicación }\end{array}$} & \multicolumn{5}{|c|}{ Años de observación } \\
\cline { 2 - 6 } & $\begin{array}{c}1987 \\
(1 \text { año })\end{array}$ & $\begin{array}{c}1988 \\
(2 \text { años })\end{array}$ & $\begin{array}{c}1989 \\
(3 \text { años })\end{array}$ & $\begin{array}{c}1990 \\
(4 \text { años })\end{array}$ & $\begin{array}{c}1991 \\
(5 \text { años })\end{array}$ \\
\hline Semanal & $1-52$ & $1-104$ & $1-156$ & $1-208$ & $1-260$ \\
\hline Quincenal & $1-26$ & $1-52$ & $1-78$ & $1-104$ & $1-130$ \\
\hline Mensual & $1-12$ & $1-24$ & $1-36$ & $1-48$ & $1-60$ \\
\hline Bimestral & $1-6$ & $1-12$ & $1-18$ & $1-24$ & $1-30$ \\
\hline Trimestral & $1-4$ & $1-8$ & $1-12$ & $1-16$ & $1-20$ \\
\hline Cuatrimestral & $1-3$ & $1-6$ & $1-9$ & $1-12$ & $1-15$ \\
\hline Semestral & $1-2$ & $1-4$ & $1-6$ & $1-8$ & $1-10$ \\
\hline Anual & 1 & $1-2$ & $1-3$ & $1-4$ & $1-5$ \\
\hline
\end{tabular}

\begin{tabular}{|c|c|c|c|c|c|c|}
\hline \multicolumn{7}{|c|}{$\begin{array}{l}\text { Tabla } 2 \\
\begin{array}{l}\text { Distribución de las revistas de sociología en cada categoría por } \\
\text { frecuencia de publicación y años de participación }\end{array}\end{array}$} \\
\hline \multirow{2}{*}{$\begin{array}{l}\text { Frecuencia de } \\
\text { Publicación }\end{array}$} & \multicolumn{6}{|c|}{ Años de participación } \\
\hline & 1 año & 2 años & 3 años & 4 años & 5 años & Total \\
\hline Mensual & & & & & 1 & 1 \\
\hline Bimestral & & & & 1 & 7 & 8 \\
\hline Trimestral & 1 & 1 & & 2 & 55 & 59 \\
\hline Cuatrimestral & & & & & 4 & 4 \\
\hline Semestral & & & & 3 & 5 & 8 \\
\hline Anual & 1 & 1 & 2 & 4 & 10 & 18 \\
\hline Total & 2 & 2 & 2 & 10 & 82 & 98 \\
\hline
\end{tabular}

\begin{tabular}{|c|c|c|c|c|c|c|}
\hline \multicolumn{7}{|c|}{$\begin{array}{c}\text { Tabla } 3 \\
\text { Distribución de la productividad total de las revistas de sociología } \\
\text { en cada categoría según sus frecuencias de publicación y años } \\
\text { de participación }\end{array}$} \\
\hline \multirow{2}{*}{$\begin{array}{l}\text { Frecuencia de } \\
\text { Publicación }\end{array}$} & \multicolumn{6}{|c|}{ Años de participación } \\
\hline & 1 año & 2 años & 3 años & 4 años & 5 años & Total \\
\hline Mensual & & & & & $\begin{array}{l}145 \\
(1) \\
\end{array}$ & $\begin{array}{l}145 \\
(1)\end{array}$ \\
\hline Bimestral & & & & $\begin{array}{l}67 \\
(1)\end{array}$ & $\begin{array}{l}1321 \\
(7)\end{array}$ & $\begin{array}{l}1388 \\
(8)\end{array}$ \\
\hline Trimestral & $\begin{array}{l}16 \\
(1)\end{array}$ & $\begin{array}{l}15 \\
(1)\end{array}$ & & $\begin{array}{l}69 \\
(2) \\
\end{array}$ & $\begin{array}{l}6563 \\
(55) \\
\end{array}$ & $\begin{array}{l}6663 \\
(59) \\
\end{array}$ \\
\hline Cuatrimestral & & & & & $\begin{array}{l}333 \\
(4) \\
\end{array}$ & $\begin{array}{l}333 \\
(4) \\
\end{array}$ \\
\hline Semestral & & & & $\begin{array}{l}144 \\
(3)\end{array}$ & $\begin{array}{l}426 \\
(5)\end{array}$ & $\begin{array}{l}570 \\
(8)\end{array}$ \\
\hline Anual & $\begin{array}{l}12 \\
(1)\end{array}$ & $\begin{array}{l}25 \\
(1)\end{array}$ & $\begin{array}{l}57 \\
(2)\end{array}$ & $\begin{array}{l}154 \\
(4)\end{array}$ & $\begin{array}{l}605 \\
(10)\end{array}$ & $\begin{array}{l}853 \\
(18)\end{array}$ \\
\hline Total & $\begin{array}{l}28 \\
(2)\end{array}$ & $\begin{array}{l}40 \\
(2)\end{array}$ & $\begin{array}{l}57 \\
(2)\end{array}$ & $\begin{array}{l}434 \\
(10)\end{array}$ & $\begin{array}{l}9393 \\
(82)\end{array}$ & $\begin{array}{l}9952 \\
(98)\end{array}$ \\
\hline
\end{tabular}

Community Sociology comenzaron a ser publicadas en 1990, y así sucesivamente. Sin embargo, de esas 115 revistas, 15 títulos fueron cortados por diferentes razones. Algunas ya habían cesado en su publicación, mientras que otras no habían sido publicadas regularmente en los últimos tres años; entonces, había posibilidades de que no continuaran siendo publicadas. Dos de ellas habían cambiado sus títulos, pero continuaban siendo publicadas por la misma institución; en este caso, la productividad fue acumulada al nuevo título. Esta depuración complementaria produjo una lista final de 98 revistas de sociología que es objeto de análisis en esta investigación. La distribución de frecuencias puede ser observada en la Tabla 2.

De 1987 a 1991, esas 98 revistas publicaron conjuntamente 1643 fascículos y produjeron 9952 artículos. La productividad media en el periodo fue de 6.06 artículos por fascículo y 102.3 artículos por cada revista. Fue calculada la productividad total para cada célula de esta distribución. Como puede observarse en la Tabla 3, de los 9952 artículos producidos, 94.4\% (9 393) de los artículos fueron producidos por revistas con una tasa de participación de cinco años; 4.4\% (434) artículos por aquellas que participaron con cuatro años; $0.59 \%$ (57) con tres años; $0.4 \%$ (40) con dos años; y $0.3 \%$ (28) por aquellas revistas que participaron nada más con un año.

Aquellas revistas que participaron con uno, dos y tres años conjuntamente produjeron solamente $1.25 \%$ de los artículos. Por otro lado, una única revista de frecuencia mensual produjo $1.5 \%$ de los artículos; $13.9 \%$ de los artículos fueron producidos por las revistas bimestrales; $66.9 \%$ por las cuatrimestrales; $3.4 \%$ por las trimestrales; $6.2 \%$ por las semestrales; y $8.1 \%$ por las anuales. Basado en el número de fascículos que se esperaban que fuesen publicados por cada revista (de acuerdo con sus años de participación y frecuencia de publicación), fue calculada la productividad media para cada célula. Los resultados son mostrados en la Tabla 4. Las revistas anuales y semestrales fueron relativamente las más productivas que el resto. Obsérvese también que existe poca diferencia en la productividad de las revistas trimestrales, cuatrimestrales y bimestrales. La única 


\begin{tabular}{|c|c|c|c|c|c|c|}
\hline \multicolumn{7}{|c|}{$\begin{array}{l}\text { Tabla } 4 \\
\text { Productividad media de las revistas de sociología en cada categoría según el } \\
\text { número de fascículos publicados }\end{array}$} \\
\hline \multirow{2}{*}{$\begin{array}{l}\text { Frecuencia de } \\
\text { Publicación }\end{array}$} & \multicolumn{6}{|c|}{ Años de participación } \\
\hline & 1 año & 2 años & 3 años & 4 años & 5 años & Total \\
\hline Mensual & & & & & 2.4 & 2.4 \\
\hline Bimestral & & & & 2.8 & 6.3 & 5.9 \\
\hline Trimestral & 4.0 & 1.9 & & 2.2 & 6.0 & 5.8 \\
\hline Cuatrimestral & & & & & 5.6 & 5.6 \\
\hline Semestral & & & & 6.0 & 8.5 & 7.7 \\
\hline Anual & 12.0 & 12.5 & 9.5 & 9.6 & 12.1 & 11.4 \\
\hline Total & 5.6 & 4.0 & 9.5 & 4.5 & 6.1 & 6.0 \\
\hline
\end{tabular}

Tabla 5

Productividad estimada de las revistas según los años de participación

\begin{tabular}{||l|l|l|l|l|l|l||}
\hline $\begin{array}{l}\text { Años de } \\
\text { Particip. }\end{array}$ & $\begin{array}{c}\text { No. de } \\
\text { Revistas }\end{array}$ & $\begin{array}{c}\% \text { de } \\
\text { Revistas }\end{array}$ & $\begin{array}{c}\text { Prod. } \\
\text { Total }\end{array}$ & $\begin{array}{c}\% \text { de } \\
\text { Prod.Total }\end{array}$ & $\begin{array}{c}\text { Prod. } \\
\text { Media }\end{array}$ & $\begin{array}{c}\text { \% de Prod. } \\
\text { Media }\end{array}$ \\
\hline 1 & 2 & 2.0 & 28 & 0.3 & 14 & 6.3 \\
\hline 2 & 2 & 2.0 & 40 & 0.4 & 20 & 9.0 \\
\hline 3 & 2 & 2.0 & 57 & 0.6 & 28.5 & 13.2 \\
\hline 4 & 10 & 10.3 & 434 & 4.4 & 43.4 & 19.5 \\
\hline 5 & 82 & 83.7 & 9393 & 94.3 & 114.5 & 52.0 \\
\hline Total & 98 & 100.0 & 9952 & 100.0 & 220.4 & 100.0 \\
\hline
\end{tabular}

revista mensual fue relativamente la menos productiva de todas ellas. Además, la productividad media tiende a aumentar conforme aumentan los años de participación de cada frecuencia de publicación con excepción de las anuales. Esta diferencia en las revistas anuales puede ser consecuencia de la cantidad de revistas que participaron con uno y dos años, en este caso solamente uno, por eso, su productividad media por fascículo fue igual a su productividad total.

La Tabla 5 muestra la distribución de la productividad estimada de acuerdo con los años de participación. Obsérvese que hay un incremento constante en la proporción de aquellas revistas participantes con más años, haciendo evidente que existe una relación monotónica entre productividad y años de participación. La correlación encontrada fue de 0.7301 cuando los años de participación fueron referidos a la productividad total. Esta correlación es de 0.8678 cuando es referida a la productividad media. En conclusión, se verificó una alta correlación entre la productividad de las revistas y el número de años con que cada revista participó en el periodo de la investigación. Cuando el modelo de regresión simple fue ejecutado, el $r^{2}$ ajustado fue de 0.377 . Esto significa que $38 \%$ de las variaciones que ocurrieron en la productividad (variable dependiente) dependieron del número de años con que cada revista participó en el periodo de la investigación (Variable independiente).

Se encontró también que el estadístico F1 fue igual a 3.42428 al .1616 nivel de significancia. Esto quiere decir que la probabilidad de malinterpretar este modelo es solamente de $16 \%$. El coeficiente de regresión estimado de la productividad total frente a los años de participación fue de 1912.4 y la estimación del parámetro lineal fue de -3746.8. Esos coeficientes siguen la ecuación:

$$
P=-3745.8+1912.4 Y
$$

Donde $P$ se refiere a la productividad total e $Y$ representa los años de participación de las revistas en el periodo de la investigación. Esta ecuación implica que existe una relación positiva de causa-efecto del número de años con que cada revista participó en el periodo de la investigación y su productividad total. El estadístico $t$-student fue igual a $1.850 \mathrm{y}$ -1.093 al .1613 y .3543 nivel de significancia, respectivamente. Esto confirma los resultados expresados por el estadístico $F$ explicado anteriormente.

Cuando se ejecutó una regresión simple del número de años de participación frente a la media de productividad, la $r^{2}$ ajustada encontrada fue de .67074 , indicando que $67 \%$ de las variaciones de la productividad media dependen de las variaciones del número de años con que cada revista participa en el periodo de la investigación. El estadístico F encontrado fue de 9.14836 al .0566 nivel de significancia. Esto indica que la probabilidad de malinterpretar estos resultados es solamente de $6 \%$. El coeficiente de regresión estimado de la productividad total frente a los años de participación fue 22.44 y la estimación del parámetro lineal fue de -23.24. Estos coeficientes fueron escritos como la ecuación:

$$
P=-23.24+22.44 Y
$$

Donde $P$ representa la productividad media e $Y$ el número de años con que cada revista participó en el periodo de la investigación. Esta ecuación nuevamente muestra una relación positiva de dependencia causal del número de años de participación en relación con sus productividades. El estadístico $t$-student asociado al coeficiente de regresión sugiere solamente $6 \%$ de posibilidades de error.

La Tabla 6 presenta la distribución de la productividad estimada de acuerdo con la frecuencia de publicación. También muestra el número de fascículos publicados de acuerdo con esta frecuencia de publicación y la media de fascículos publicados por revista.

Nuevamente, se notó un incremento constante en la proporción de aquellas revistas que participaron con una mayor frecuencia de publicación. Esto hace 


\begin{tabular}{|c|c|c|c|c|c|c|c|c|}
\hline \multicolumn{9}{|c|}{$\begin{array}{c}\text { Tabla } 6 \\
\text { Productividad estimada de las revistas según la } \\
\text { frecuencia de publicación }\end{array}$} \\
\hline $\begin{array}{c}\text { Frecuencia de } \\
\text { publicación }\end{array}$ & $\begin{array}{l}\text { No. de } \\
\text { Rev. }\end{array}$ & $\begin{array}{l}\% \text { de } \\
\text { Rev. }\end{array}$ & $\begin{array}{l}\text { No. de } \\
\text { Fasc. }\end{array}$ & $\begin{array}{l}\text { Prod. } \\
\text { Total }\end{array}$ & $\begin{array}{l}\% \text { de } \\
\text { Prod. }\end{array}$ & $\begin{array}{l}\text { Media } \\
\text { de Fasc. }\end{array}$ & $\begin{array}{l}\text { Media } \\
\text { de Prod. }\end{array}$ & $\begin{array}{l}\% \text { Med. } \\
\text { de Prod. }\end{array}$ \\
\hline Mensual & 1 & 1.0 & 60 & 145 & 1.5 & 60 & 145.0 & 22.8 \\
\hline Bimestral & 8 & 8.2 & 234 & 1388 & 13.9 & 29 & 173.5 & 27.4 \\
\hline Trimestral & 59 & 60.2 & 1144 & 663 & 67.0 & 19 & 112.9 & 17.8 \\
\hline Cuatrimes. & 4 & 4.0 & 60 & 333 & 3.3 & 15 & 83.3 & 13.2 \\
\hline Semestral & 8 & 8.2 & 74 & 570 & 5.7 & 9 & 71.3 & 11.2 \\
\hline Anual & 18 & 18.4 & 71 & 853 & 8.6 & 4 & 47.4 & 7.5 \\
\hline Total & 98 & 100.0 & 1643 & 9952 & 100.0 & & 633.4 & 100.0 \\
\hline \multicolumn{8}{|c|}{$\begin{array}{c}\text { Tabla } 7 \\
\begin{array}{c}\text { Productividad de las revistas según los años de participación y distribución } \\
\text { por zonas en el modelo Bradford clásico }\end{array}\end{array}$} & \\
\hline \multirow{2}{*}{\multicolumn{2}{|c|}{ Zonas }} & \multicolumn{6}{|c|}{ Años de participación } & \\
\hline & & 5 & 4 & 3 & & 2 & 1 & \\
\hline \multicolumn{2}{|l|}{1} & $\begin{array}{l}3326 \\
(14)\end{array}$ & & & & & & \\
\hline \multicolumn{2}{|l|}{2} & $\begin{array}{l}3299 \\
(25)\end{array}$ & & & & & & \\
\hline \multicolumn{2}{|l|}{3} & $\begin{array}{l}2768 \\
(43)\end{array}$ & $\begin{array}{l}434 \\
(10) \\
\end{array}$ & $\begin{array}{l}57 \\
(2)\end{array}$ & $\begin{array}{l}40 \\
(2\end{array}$ & & $\begin{array}{l}28 \\
(2)\end{array}$ & \\
\hline \multicolumn{2}{|c|}{ Total } & $\begin{array}{l}9393 \\
(82)\end{array}$ & $\begin{array}{l}434 \\
(10)\end{array}$ & $\begin{array}{l}57 \\
(2)\end{array}$ & $\begin{array}{l}40 \\
(2\end{array}$ & & $\begin{array}{l}28 \\
(2)\end{array}$ & \\
\hline
\end{tabular}

evidente que existe una relación monotónica entre la frecuencia de publicación y la productividad. La correlación encontrada fue de 0.9966 cuando la frecuencia de publicación, expresada como el número de fascículos producidos en el periodo de investigación, es referida a la productividad total. Esta correlación es de 0.7504 cuando la relación es establecida entre la media de fascículos producidos y la productividad media de acuerdo con la frecuencia de publicación. En conclusión, existe una alta correlación entre la frecuencia de publicación y la productividad de las revistas. Cuando el modelo de regresión simple fue ejecutado, el $r^{2}$ ajustado fue de 0.99156 . Esto significa que $99.2 \%$ de las variaciones que ocurrieron en la productividad (variable dependiente) dependieron de las variaciones ocurridas en la frecuencia de publicación (Variable independiente). El estadístico F encontrado fue de 588.65131 al .0000 nivel de significancia, lo que denota que la probabilidad de malinterpretar este modelo estadístico es igual al cero por ciento. El coeficiente de las variaciones del número de fascículos publicados por cada revista. El estadístico F encontrado fue de 5.15513 al .0857 nivel de significancia. Esto expresa que la probabilidad de malinterpretar estos resultados es solamente de $8.6 \%$. Estos coeficientes fueron escritos como la ecuación:

$$
P=65.589092+1.763717 S
$$

Donde $P$ representa la productividad media y $S$ el número medio de fascículos publicados por cada revista en el periodo de la investigación. Esta ecuación nuevamente muestra una relación positiva de dependencia causal entre el promedio de fascículos publicados por cada revista y sus productividades. El estadístico $t$-student asociado al coeficiente de regresión sugiere solamente $8.6 \%$ de posibilidades de error.

Para hacer una comparación con la aplicación del modelo clásico de Bradford, fue ejecutada una división por zonas de las 98 revistas estudiadas. Los valores esperados fueron 14.0, 29.4, y 61.74 en las zonas 1 , 2, y 3, respectivamente. Esta distribución se ajustó adecuadamente a los valores observados de 14, 25, y 59, respectivamente. Sin embargo, como puede ser observado en la Tabla 7, absolutamente todas las revistas de las zonas 1 y 2 habían participado con 5 años en el periodo de la investigación. Las revistas que participaron con menos años se agrupaban en la zona 3. La productividad media por zona fue también de 238 (zona 1), 132 (zona 2) y de 56.4 (zona 3). No obstante, la productividad media de acuerdo con el número de fascículos producidos en ese mismo periodo fue de 10.7 (zona 1), 5.9 (zona 2) y 4.2 artículos (zona 3), respectivamente.

La distribución zonal del modelo Bradford clásico de acuerdo con la frecuencia de publicación puede ser visto en la Tabla 8. Las revistas más frecuentemente publicadas (mensuales, bimestrales y trimestrales) se agruparon en las zonas $1 \mathrm{y}$ 2. Aquellas revistas publicadas con menos frecuencia se agruparon en la zona 3. Como tendencia, las revistas más frecuentemente publicadas se dispersaron en los rangos más elevados y las revistas con menos frecuencia de publicación ocuparon los rangos más bajos. En resumen, parece claro que tanto el número de años con que determinada revista participa en el periodo de la investigación y la fre- 


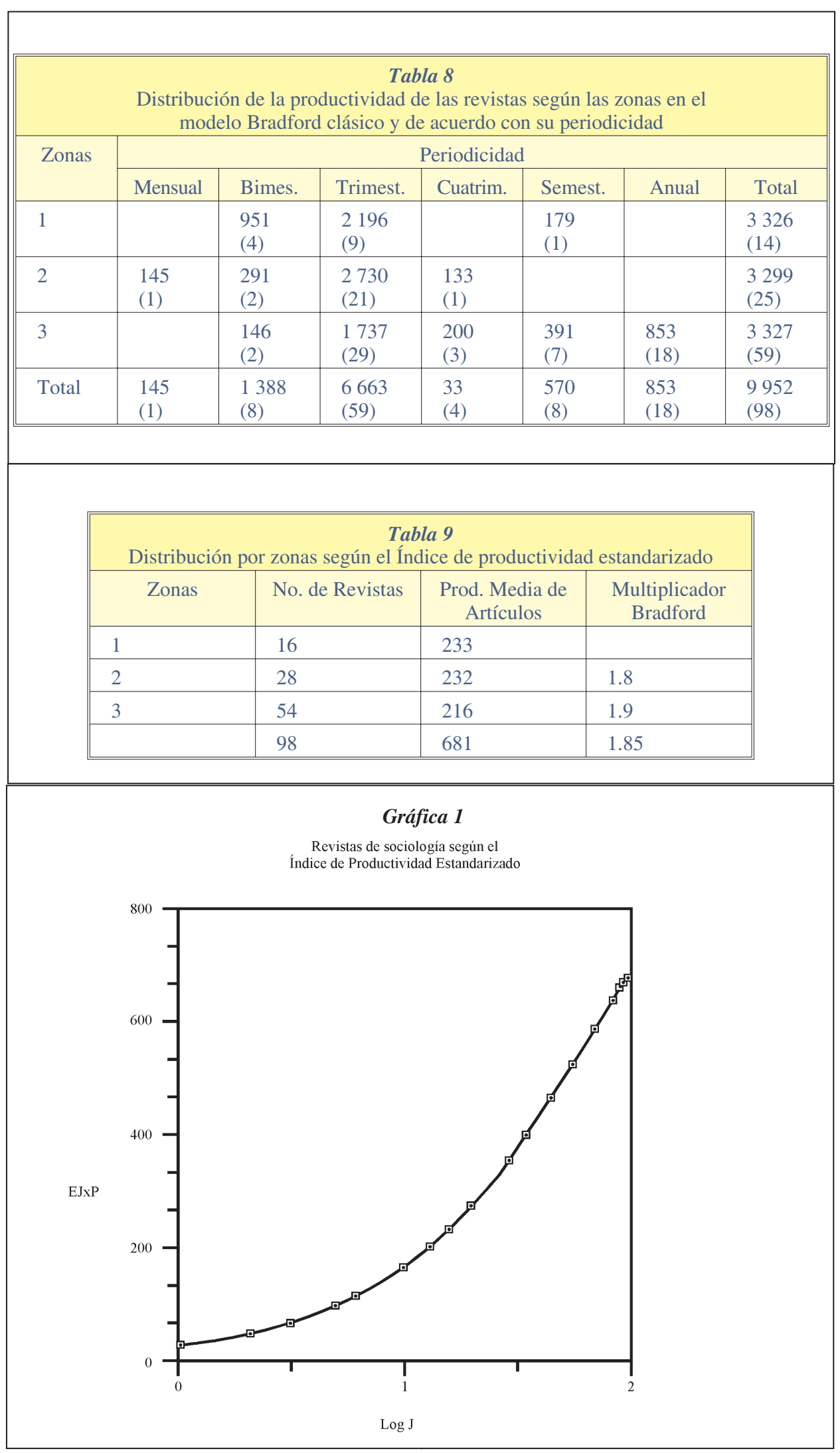

cuencia de sus publicaciones, influyen en el rango de las revistas al tope o al final de la distribución de Bradford. Las revistas más frecuentemente publicadas y con más años de participación tienden a de la distribución. En consecuencia, puede concluirse que la ley de Bradford en su versión clásica es un mal indicador de la productividad de las revistas.

Tal vez sería interesante probar un modelo alternativo que tome en consideración ambas variables. Con esta idea fue realizada una división por zonas basada en el Índice de Productividad Estándar (IPE). Este IPE es solamente una productividad media estimada para cada revista de acuerdo con el número de fascículos producidos en el periodo de la investigación, por eso, el número de años de participación está implícitamente incluido. La fórmula usada fue:

$$
I P E=\frac{\sum A_{j}}{\sum F_{n}}
$$

Donde

$\sum A_{j}=$ Total de artículos producidos en el periodo de la investigación por una determinada revista.

$\sum F_{n}=$ Número de fascículos esperados que una determinada revista produzca de acuerdo con el número de años con que participa en el periodo de la investigación y su frecuencia de publicación.

Usando esta fórmula, el Índice de Productividad Estandarizado (IPE) para cada revista fue calculada de la forma siguiente: para Journal of Marriage and the Family (491/20 = 24.6); para American Sociological Review $(332 / 30=11.1)$; para Family Relations $(290 / 20=14.5)$; para Teaching Sociology $(258 / 20=12.9)$; y así sucesivamente. Cuando esta distribución fue comparada con la distribución clásica del modelo Bradford, el IPE produjo modificaciones sustanciales en el rango de las revistas. Por eso, usando el IPE, se efectuó una nueva distribución por zonas. Como se puede observar en la Tabla 9, esta nueva división produjo 3 zonas donde las 16 revistas más productivas (zona 1) en media produjeron 14.6 artículos por fascículo; los productores medios (zona 2) 8.3 artículos; mientras que los pequeños productores (zona 3 ) fueron responsables de sólo 4.0 artículos por fascículo. Los valores observados de 16, 28 y 54 están bien próximos a los valores esperados de $16,29.6$ y 54.8 para las zonas 1, 2, y 3, respectivamente. El ajuste a $1: n: n^{2}$ es exacto.

La Grafica 1 muestra el trazado para esta nueva división. Se puede observar la 


\begin{tabular}{|c|c|c|c|c|c|c|c|}
\hline \multirow{3}{*}{ Zonas } & \multicolumn{6}{|c|}{$\begin{array}{c}\text { Tabla } 10 \\
\text { Distribución de las revistas por zonas, frecuencia de publicación y } \\
\text { sus productividades de acuerdo con el IPE }\end{array}$} & \\
\hline & \multicolumn{7}{|c|}{ Periodicidad } \\
\hline & Mensual & Bimes. & Trimest. & Cuatrim. & Semest. & Anual & Total \\
\hline 1 & & $\begin{array}{l}11 \\
(1)\end{array}$ & $\begin{array}{l}65 \\
(4)\end{array}$ & & $\begin{array}{l}18 \\
(1)\end{array}$ & $\begin{array}{l}139 \\
(10)\end{array}$ & $\begin{array}{l}233 \\
(16)\end{array}$ \\
\hline 2 & & $\begin{array}{l}16 \\
(2)\end{array}$ & $\begin{array}{l}127 \\
(16)\end{array}$ & $\begin{array}{l}9 \\
(1)\end{array}$ & $\begin{array}{l}24 \\
(3)\end{array}$ & $\begin{array}{l}56 \\
(6)\end{array}$ & $\begin{array}{l}232 \\
(28)\end{array}$ \\
\hline 3 & $\begin{array}{l}2 \\
(1)\end{array}$ & $\begin{array}{l}19 \\
(5)\end{array}$ & $\begin{array}{l}151 \\
(39)\end{array}$ & $\begin{array}{l}13 \\
(3)\end{array}$ & $\begin{array}{l}18 \\
(4)\end{array}$ & $\begin{array}{l}10 \\
(2)\end{array}$ & $\begin{array}{l}213 \\
(54)\end{array}$ \\
\hline Total & $\begin{array}{l}2 \\
(1)\end{array}$ & $\begin{array}{l}46 \\
(8)\end{array}$ & $\begin{array}{l}389 \\
(59)\end{array}$ & $\begin{array}{l}22 \\
(4)\end{array}$ & $\begin{array}{l}60 \\
(8)\end{array}$ & $\begin{array}{l}205 \\
(18)\end{array}$ & $\begin{array}{l}678 \\
(98)\end{array}$ \\
\hline
\end{tabular}

curva clásica que después se transforma en una línea recta para terminar con una caída final similar a las gráficas bradfordianas ya conocidas. La Tabla 10 muestra la distribución del IPE por zonas y frecuencia de publicación. Obsérvese que la dispersión de las revistas por todas las células es notoria, significando que la medida usada para evaluar la productividad es la misma y homogénea para todas las revistas. Cuando se hizo un análisis comparativo entre las revistas agrupadas por zonas entre el modelo Bradford clásico (Tabla 7) y el Índice de Productividad estandarizado (Tabla 9), se obtuvieron los siguientes resultados: de las 14 revistas agrupadas en la zona 1 en la distribución bradfordiana clásica, solamente $6(42 \%)$ de ellas se mantuvieron en esta agrupación; $5(36 \%)$ descendieron a la zona 2 ; y $2(22 \%)$ descendieron a la zona 3 . Fenómeno similar ocurrió con las 25 revistas de la zona 2, donde solamente $12(48 \%)$ de ellas permanecieron en esa zona 2 , y $13(52 \%)$ revistas descendieron a la zona 3. Contrariamente, de las 59 revistas agrupadas en la zona 3, 10 revistas (17\%) pasaron a la zona $1 ; 9(15 \%)$ subieron a la zona 2, y las $40(68 \%)$ revistas restantes conservaron su rango.

Siguiendo el modelo Bradford, en el Anexo 1, las revistas analizadas en este artículo han sido ordenadas en forma decreciente de las más a las menos productivas en artículos de acuerdo con sus IPEs. Por ejemplo, entre 1987-1991, la revista Journal of Marriage and the Family produjo 24.6 artículos por fascículo; así, ésta fue la más productiva. Annual Review of Sociology produjo 20.4 artículos por fascículo; Free Inquiry in Creative Sociology produjo 17.9 artículos; Studies in Latin American Popular Culture produjo 16.6; Family Relations, 13.3, colocándose en segundo, tercero y cuarto lugares, y así sucesivamente.

\section{CONCLUSIÓN}

Como se ha afirmado orginalmente, la literatura sobre la ley de Bradford es extensa. La mayor parte de esta literatura está dedicada a la discusión de la forma de la curva, el ajustamiento al modelo matemático, etcétera. Sin embargo, se ha prestado poca atención al hecho de que la productividad de una revista puede ser influida por su frecuencia de publicación y los años con que participa en el periodo de la investigación. Más aún, el hecho de que esas dos variables pueden ser la causa de que una revista se coloque al tope o al final de una distribución del tipo de Bradford, no ha sido investigado. Como se dijo en la hipótesis, esta investigación ha demostrado que esto es verdad para las revistas americanas del área de sociología que produjeron artículos entre los años 1987 y 1991. Estos resultados indican que la ley de Bradford, en su forma clásica, no es adecuada para la selección de revistas dedicadas a este campo, pues excluye aquellas revistas que participan con menos años y con menos frecuencia de publicación. También el modelo superestima la productividad de aquellas revistas más frecuentemente publicadas y que participan con más años. Sin embargo, cuando el IPE es usado como una base alternativa para la aplicación de la distribución Bradford, parece identificar las revistas más dedicadas al asunto y también medir más adecuadamente su productividad. Esto parece ser consecuencia de que el IPE controla mejor la frecuencia de publicación y los años de participación en el periodo de investigación, variables a las que ni Bradford ni los bradfordianos hasta ahora han prestado atención. Tal vez, el problema no sea el modelo sino la forma como ésta ha sido usada hasta el momento. Sin embargo, creo que son necesarias más investigaciones empíricas para entender mejor este fenómeno.

\section{BIBLIOGRAFÍA}

AIYEPEKU, W. O. "Bradford distribution theory : compounding of Bradford periodical literature in geography”. Journal of documentation, 33:210-217, 1977.

BAGUENA CERVELLERA, María José. "La microbiología en los artículos de revistas y comunicaciones a congresos de medicina del siglo xix español”. Revista Española de Documentación Científica, 7(1):29-38, 1984.

BASU, Aparna. "Hierarchical distributions and Bradford's law". Journal of the American Society for Information Science, 43(7):494-500, August 1992.

BRADFORD, B. S. "Documentation". Washington, D.C. : Public Affairs Press, 1950. p. 106-121.

_ _. "Sources of information on specific subjects". Engineering, 137:85-86, Jan. 26, 1934. 
BROOKES, B. C. "Bradford's law and the bibliography of science". Nature, 224(5523):953-956, Dec. 1969.

BROOKES, B. C. "Theory of Bradford Law". Journal of documentation, 33(3):180-209, 1977.

BULICK, S. "Book use as a Bradford-Zipf phenomenon". College and Research Libraries, 39:215-219, May 1978.

CALDEIRA, P. da T. "Dispersão e produtividade da literatura sobre doenças de chagas". Revista de Biblioteconomia de Brasilia, 3(2):133-142, Jul.-Dez. 1975.

DePEW, John N. \& Santi Basu. "The application of Bradford's law in selecting periodicals on conservation and preservation of library materials". Collection Management, 8(1):55-65, Spring 1986.

FIGUEIREDO, L. M. de. "Distribuição da literatura geológica brasileira : un estudo bibliométrico". Ciência da Informação, Rio de Janeiro, 2(1):27-40, 1973.

GOFFMAN, W. \& T. G. Morris. "Bradford's Law and library acquisitions". Nature, 226:922-923, June 6, 1970.

KENDALL, M.G. "The bibliography of operations research". Operations Research Quarterly, 11 (1/2):31-36, March-June 1960.

LAWANI, S.M. "Bradford's law and the literature of agriculture". International library review, 5:341-350, July 1973.

OLUIC-VUKOVIC, Vesna. "Why has Bradford's law been an ellusive phenomenon so for?". Journal of the American Society for Information Science, 44(3):182-183, April 1993.

PAO, Miranda Lee. "Bibliometrics and computational musicology". Collection management, 3(1):97-109, Spring 1979.

PINHEIRO, Lena Vania Ribeiro. "Lei de Bradford : uma reformulação conceitual”. Rio de Janeiro : UFRJ/IBICT, 1982. Thesis (MLS).

QIU, Liwen. "An empirical examination of the existing models for Bradford's law”. Information Processing \& Management, 26(5):655-672, 1990.

QUEIROZ, S. de S. "Bibliografia brasielira de botânica, 1971-1972 : un estudo bibliomêtrico". Ciência da Informação, Rio de Janeiro, 4(1):55-66, 1975.

SIVERS, Robert. "Partitioned Bradford ranking and the serials problem in Academic Research libraries". Collection building, 8(2):12-19, 1987.

ULRICH's International Periodicals Directory, 1993-1994 : including irregular serials \& annuals. 32nd ed. New York : R. R. Bowker, 1993.

URBIZAGÁSTEGUI ALVARADO, Rubén. "Concentração e dispersão da literatura nos periódicos brasileiros da área de educação". Estudos avançados em Biblioteconomia e Ciência da Informação, Brasilia, 4:91-114, 1985.

_ _ _ . "Lista básica de publicações periódicas na área de educaço". Cadernos de Pesquisa, São Paulo, 44:81-89, Fev. 1983.

VICKERY, B. C. "Bradford's law of scattering". Journal of Documentation, 4(1):198-203, June 1948.

\section{Anexo 1}

\section{Revistas americanas de sociología}

\begin{tabular}{||l|c|l||}
\hline \hline Orden & IPE & \multicolumn{1}{c||}{ Título de la revista } \\
\hline 1 & 24.6 & Journal of marriage and the family \\
\hline 2 & 20.4 & Annual review of sociology \\
\hline 3 & 17.9 & Free inquiry in creative sociology \\
\hline 4 & 17.4 & Studies in symbolic interaction \\
\hline 5 & 16.6 & Studies in Latin American popular culture \\
\hline 6 & 14.5 & Family relations \\
\hline 7 & 13.3 & Research in the sociology of organizations \\
\hline 8 & 12.9 & Teaching sociology \\
\hline 9 & 12.8 & Clinical sociology review \\
\hline 10 & 12.5 & Advances in medical sociology \\
\hline 11 & 12.4 & Social forces \\
\hline
\end{tabular}

\begin{tabular}{||c|c|l||}
\hline \hline Orden & IPE & \multicolumn{1}{c||}{ Título de la revista } \\
\hline 12 & 12.0 & Research in community sociology \\
\hline 13 & 11.8 & Current perspectives in social theory \\
\hline 14 & 11.4 & Research in social movements, conflicts and change \\
\hline 15 & 11.2 & Research in social stratification and mobility \\
\hline 16 & 11.1 & American sociological review \\
\hline 17 & 10.0 & Sociological practice \\
\hline 18 & 9.8 & Sociology and social research \\
\hline 19.5 & 9.7 & Sociological forum \\
\hline 19.5 & 9.7 & Comparative social research \\
\hline 21 & 9.3 & Research in sociology of education and socialization \\
\hline 22 & 9.0 & Advances in group processes \\
\hline
\end{tabular}


Anexo 1

(Cont.)

\section{Revistas americanas de sociología}

\begin{tabular}{|c|c|c|}
\hline Orden & IPE & Título de la revista \\
\hline 23 & 8.9 & Journal of comparative family studies \\
\hline 24 & 8.8 & Sociological quarterly \\
\hline 25.5 & 8.7 & Sociological theory \\
\hline 25.5 & 8.7 & Social service review \\
\hline 27.5 & 8.6 & Berkeley journal of sociology \\
\hline 27.5 & 8.6 & Journal of sociology and social welfare \\
\hline 29 & 8.5 & California sociologist \\
\hline 30 & 8.0 & Journal of political \& military sociology \\
\hline 31 & 7.8 & Social psychology quarterly \\
\hline 32.5 & 7.6 & The American journal of sociology \\
\hline 32.5 & 7.6 & Sociological analysis \\
\hline 34.5 & 7.5 & Society \\
\hline 34.5 & 7.5 & Human organization \\
\hline 36 & 7.4 & The American sociologist \\
\hline 38 & 7.2 & Journal of health and social behavior \\
\hline 38 & 7.2 & Review of religious research \\
\hline 38 & 7.2 & Rural sociology \\
\hline 40.5 & 6.9 & Research in the sociology of health care \\
\hline 40.5 & 6.9 & Sociological inquiry \\
\hline 42 & 6.8 & Sociology of sport journal \\
\hline 43.5 & 6.7 & Humanity \& society \\
\hline 43.5 & 6.7 & Gender and society \\
\hline 46 & 6.4 & Critical sociology \\
\hline 46 & 6.4 & Sociological perspectives \\
\hline 46 & 6.4 & Social studies of science \\
\hline 48 & 6.2 & Sociological spectrum \\
\hline 49 & 6.1 & Mid-American review of sociology \\
\hline 50 & 6.0 & $\begin{array}{l}\text { International journal of politics, culture, and } \\
\text { society }\end{array}$ \\
\hline 51 & 5.9 & The Journal of sex research \\
\hline 52 & 5.8 & Sociological focus \\
\hline 53 & 5.6 & Marriage and family review \\
\hline 54 & 5.5 & Social problems \\
\hline 55.5 & 5.4 & Work and occupations \\
\hline 55.5 & 5.4 & Thesis eleven \\
\hline 58 & 5.2 & Journal of social issues \\
\hline 58 & 5.2 & Qualitative sociology \\
\hline 58 & 5.2 & Sociology of health \& illness \\
\hline 60 & 5.1 & Theory and society \\
\hline 61.5 & 5.0 & The Jewish social work forum \\
\hline
\end{tabular}

\begin{tabular}{|c|c|c|}
\hline Orden & IPE & Título de la revista \\
\hline 61.5 & 5.0 & Sociology of education \\
\hline 63 & 4.9 & Urban affairs quarterly \\
\hline 65 & 4.6 & The Journal of social psychology \\
\hline 65 & 4.6 & Comparative studies in society and history \\
\hline 65 & 4.6 & Sociological methods \& research \\
\hline 67 & 4.5 & Human mosaic \\
\hline 68.5 & 4.4 & Social science research \\
\hline 68.5 & 4.4 & $\begin{array}{l}\text { The American journal of economics and sociol- } \\
\text { ogy }\end{array}$ \\
\hline 70 & 4.2 & Social research \\
\hline 71.5 & 4.1 & Journal of contemporary ethnography \\
\hline 71.5 & 4.1 & Social biology \\
\hline 74 & 4.0 & Research on social work practice \\
\hline 74 & 4.0 & $\begin{array}{l}\text { Urban anthropology and studies of cultural } \\
\text { systems and world economic development }\end{array}$ \\
\hline 74 & 4.0 & Contemporary jewry \\
\hline 76 & 3.9 & Cultic studies journal \\
\hline 77 & 3.8 & International journal of intercultural relations \\
\hline 78 & 3.7 & $\begin{array}{l}\text { International journal of contemporary sociol- } \\
\text { ogy }\end{array}$ \\
\hline 79.5 & 3.6 & International journal of sociology \\
\hline 79.5 & 3.6 & Social policy \\
\hline 81 & 3.2 & Social justice research \\
\hline 82.5 & 3.0 & The Journal of mathematical sociology \\
\hline 82.5 & 3.0 & The Wisconsin sociologist \\
\hline 84.5 & 2.8 & The American behavioral scientist \\
\hline 84.5 & 2.8 & Education and urban society \\
\hline 86 & 2.7 & Armed forces and society \\
\hline 87 & 2.6 & Contemporary sociology \\
\hline 88 & 2.4 & Human relations \\
\hline 89 & 2.1 & Journal of social history \\
\hline 90.5 & 2.0 & Simulation \& gaming \\
\hline 90.5 & 2.0 & Social text \\
\hline 92 & 1.9 & The Responsive community \\
\hline 93 & 1.7 & The Journal of interdisciplinary history \\
\hline 94 & 1.6 & Social theory and practice \\
\hline 95 & 1.4 & Political behavior \\
\hline 96 & 1.1 & Philosophy \& public affairs \\
\hline 97 & 1.0 & Technology and culture \\
\hline 98 & 0.7 & Human rights quarterly \\
\hline
\end{tabular}

